\title{
Age Is Not Necessarily Aging: Another Step towards Understanding the 'Clocks' That Time Aging
}

\author{
Shu-Chen Li Florian Schmiedek \\ Center for Life Span Psychology, Max Planck Institute for Human Development, Berlin, Germany
}

\author{
Key Words \\ Cognitive aging · Longitudinal design - Cross-sectional \\ design · Intraindividual differences · Interindividual \\ differences $\cdot$ Cognitive neuroscience $\cdot$ Computational \\ neuroscience
}

\begin{abstract}
Hofer and Sliwinski raise a critical concern regarding a popular practice in aging research of inferring associations among aging changes of different processes from correlations based on cross-sectional age differences. It is shown analytically that cross-sectional information drawn from age-heterogeneous samples is not suitable for answering questions about the interdependence between aging changes. While in general we agree with the problem pointed out by Hofer and Sliwinski, the two aims of this commentary are: (1) to highlight links between the specific concern and two long-standing issues confronting life-span researchers and (2) to introduce alternative approaches that are currently underutilized in gerontological research. We elaborate general issues with respect to challenges in inferring aging changes from age differences and using age as a proxy for aging. We show that several other sources of variance independent of rates of change, such as cohort- and mortalityrelated selection effects, are confounded in age-hetero-
\end{abstract}

geneous designs. We suggest general considerations for developing more explicit theories of cognitive aging in the future. Alternative research methods and paradigms for studying the causes and mechanisms of the aging mind at different time scales and levels are also highlighted. The role of chronological age in aging research is rather 'superficial'. To understand the underlying processes and mechanisms of cognitive aging, classical process-oriented, rather than solely age-oriented, theoretical conceptions need to be reconsidered and reified with new methodological and empirical advances. Concerted gerontological research endeavors utilizing recent progresses made in statistical analyses of dynamic processes and in cognitive and computational neurosciences that may lead to fruitful breakthroughs.

Copyright $@ 2002$ S. Karger AG, Basel

\section{Introduction}

A central issue in the context of life span research is to what extent valid inferences about extent, pattern, and covariation of longitudinal intraindividual aging changes can be drawn from cross-sectional interindividual age differences. Over 30 years ago, Schaie [1] pointed out the limitations of cross-sectional designs. However, research pragmatics and theoretical complexity hampered at-

\begin{tabular}{ll}
\hline KARGER & ( ) 2002 S. Karger AG, Basel \\
0304-324X/02/0481-0005\$18.50/0 \\
$\begin{array}{l}\text { Fax +4161306 1234 } \\
\begin{array}{l}\text { E-Mail karger@karger.ch } \\
\text { www.karger.com }\end{array}\end{array}$ & $\begin{array}{l}\text { Accessible online at: } \\
\text { www.karger.com/journals/ger }\end{array}$
\end{tabular}

Shu-Chen $\mathrm{Li}$

Center for Life Span Psychology

Max Planck Institute for Human Development, Lentzeallee 94

D-14195 Berlin (Germany)

E-Mail shuchen@mpib-berlin.mpg.de 


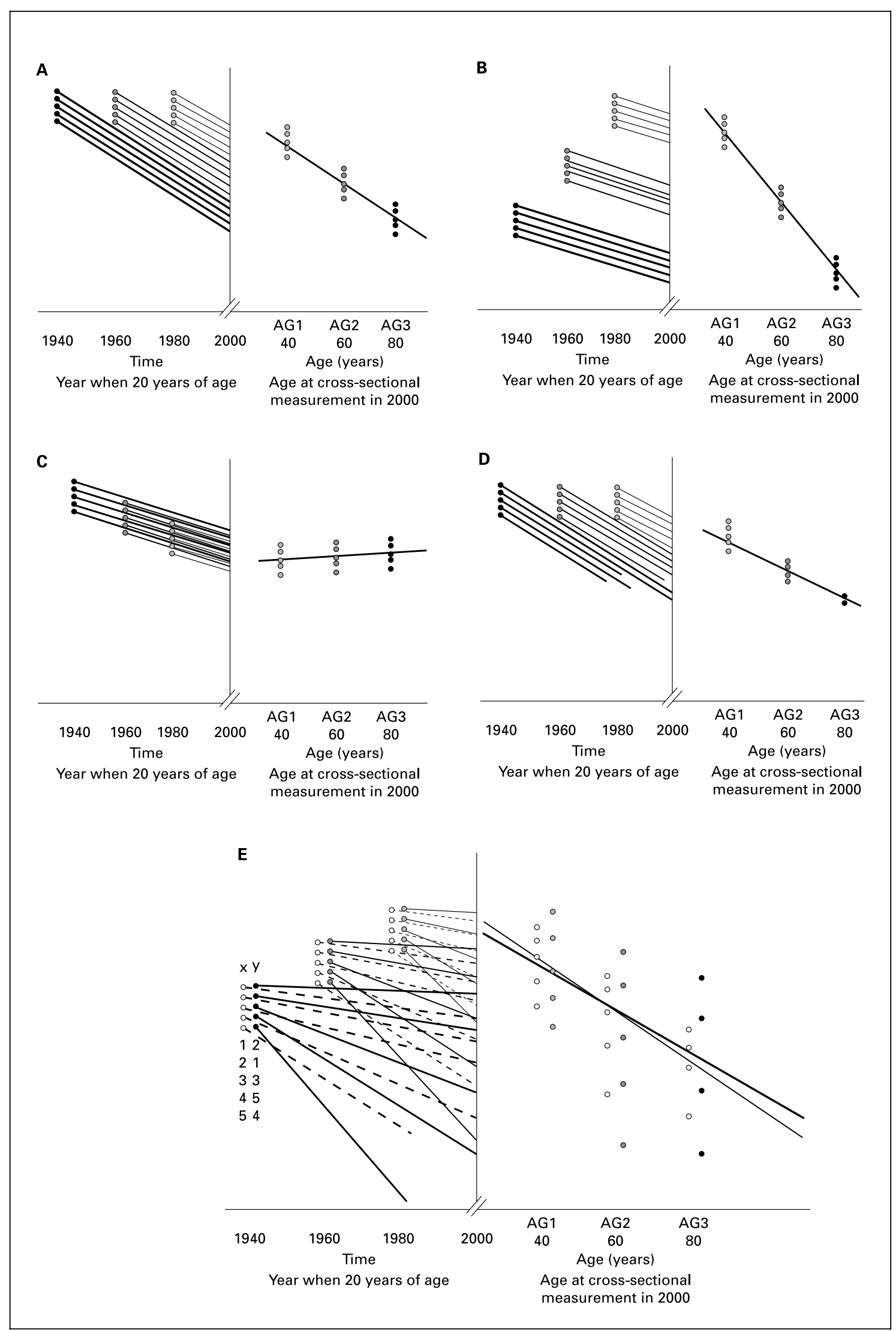


tempts to adopt longitudinal research strategies. Hofer and Sliwinski's article alerts the research community specifically to the problems of inferring the interdependence among aging changes in different processes from the associations between cross-sectional age differences.

Hofer and Sliwinski's formal analyses make a clear point about an undeniable problem: in cross-sectional age-heterogeneous designs, there is the danger of mean population age trends confounding (and even augmenting) the extent of the inferred intercorrelation between presumed rates of change reflected in the association between age differences. In general, we agree with the problem being pointed out; however, regarding its implications, we hold different views. We consider a wider spectrum of research orientations and methodologies besides those suggested in the target article. Below, we first elaborate on two long-standing problems confronting lifespan researchers: (1) inferring aging changes from crosssectional gradients based on age differences and (2) using chronological age as a proxy for development and aging. Both issues are closely related to Hofer and Sliwinski's concerns. We then discuss general considerations for constructing theories that more explicitly outline mechanisms of aging. Alternative methods and paradigms that could be adopted and adapted to address related issues and generate new research approaches are also highlighted.

Fig. 1. A-D Schematic diagrams showing subsets of possible patterns of relations between longitudinal intraindividual changes and crosssectional age gradients based on age differences. Note that linear declines are assumed in these figures. Different patterns of nonlinear changes are also possible. A Only individual differences in initial level at age 20. B Individual differences in initial level at age 20 plus cohort effect favoring younger cohorts. C Individual differences in initial level at age 20 plus cohort effect favoring older cohorts. D Individual differences in initial level at age 20 plus mortality-based negative selection effect. E The key notions in Hofer and Sliwinski's NAC and SNAC designs. The rank order of individual differences in crosssectional samples reflects more and more individual differences in rates of change in the groups with individuals who have undergone a longer period of change. Although there are cohort and mortality effects, when the correlations between variables $\mathrm{x}$ and $\mathrm{y}$ are analyzed within each narrow age group, the cohort effect is not operative. For each age group two variables, $\mathrm{x}(\mathrm{O})$ and $\mathrm{y}(\boldsymbol{O})$, are shown. The numbers denote the rank ordering of individual differences in level for each variable. The example given here shows that the rank orderings of the two variables are correlated, but not perfectly. See text for more details.

\section{Challenges to Inferring Aging Changes from Age Differences}

Besides neurobiological influences, development and aging take place in environmental-social contexts which also contribute to the processes of change. These influences and their interactions on life span development meet at the individual level. Thus, direct expressions of behavioral and cognitive changes happen first at the individual level rather than between individuals at the group level. Nonetheless, researchers often have to forego longitudinal studies and, instead, adopt cross-sectional designs with the hope that cross-sectional age differences might reasonably approximate longitudinal intraindividual aging changes.

Alas, the road of inferring aging changes from age differences is not at all straightforward; other sources of variance bend the path. To illustrate, four hypothetical univariate examples are graphically represented in figure 1 to compare and contrast a rather limited subset of possible relations between the extent of longitudinal aging changes and the approximated cross-sectional gradients based on age differences. Longitudinal aging changes across 20, 40, or 60 years for three different cohorts, starting from an assumed maturation point at age 20 , are plotted on the left side of each panel. The corresponding cross-sectional age groups sampled at a given measurement occasion (i.e., year 2000) when the individuals are, respectively, 40, 60, and 80 years old and the gradients based on interindividual age differences are plotted on the right within each panel. It would be wrong to suggest that cross-sectional age differences, given the between-individual nature, could never offer valid inferences about intraindividual aging changes. Panel A shows that when there are only interindividual differences in initial levels, and no any other appreciable effects, the cross-sectional gradient based on mean age differences of the group approximates the extent of aging change well. The world of empirical reality, however, is more challenging than this simplistic case. Other sources of variance, such as cohort and mortality-related selection effects with respect to initial levels and (or) rates of changes, and the retest effects in longitudinal designs could obscure the inferences. Given limitations of space and graphical clarity, three rather simplistic examples involving only cohort or mortality effects with respect to ability (or performance) level are illustrated here. For instance, panel B illustrates that if there were cohort effects favoring the younger cohorts in terms of initial level at the point of maturity in addition to individual differences in level, the cross-sectional gradient based on 
age differences would overestimate longitudinal aging change. In the opposite case (panel C), in which cohort effects on initial levels favor the older cohorts, cross-sectional age differences would underestimate (or estimate the effect in the opposite direction of) longitudinal aging change. Panel D shows the case when mortality-related selection effects negatively related to initial levels are involved; here cross-sectional age differences would underestimate longitudinal age change. Common discrepancies between negative cross-sectional age differences in most intellectual abilities starting at about the age of 30 [see 2 for a review] and negative longitudinal aging changes not being evident until the 7th decade of life could result from various combinations of cohort, mortality, and retest effects [see 3 for a review]. These potential influences on mean age trends, that themselves do not necessarily affect intraindividual change or interindividual differences in rates of change per se, make the task of inferring interdependence of aging changes from age differences to be rather problematic - the main concern highlighted by Hofer and Sliwinski. To circumvent these potential confounded sources of variance affecting mean age trends and, subsequently, inferences about interdependence between processes, Hofer and Sliwinski suggest that researchers use cross-sectional samples with relatively narrow age ranges.

Panel E depicts our interpretation of Hofer and Sliwinski's key arguments. On the left, it shows that there are both a cohort effect on the initial level favoring the younger cohorts and a mortality effect in that one individual from the oldest cohort died at 1980 and could not be sampled at the measurement occasion in the year 2000. On the right, it shows that if the correlation between variables $\mathrm{x}$ and $\mathrm{y}$ are based on cross-sectional age differences, the relation would reflect mean age trends that are in part due to cohort and mortality effects, in addition to the association attributable to rank orderings and individual differences in the rates of change. However, within each of the narrow age cohort (NAC) samples, interindividual differences in the rank ordering within an age group only reflect individual differences at initial level and the rate of aging change prior to the current measurement occasion. When interdependence between two (or more) change processes are approximated within each of the NAC samples, other potential effects affecting mean age trends become inconsequential, because they do not operate in this case. When comparing across a sequence of NAC samples (i.e., the SNAC design), the rank ordering of interindividual differences carries more information about individual differences in rates of change in samples with individuals who have gone through a longer time period of change. Thus, if there were some related causes affecting individual differences in the rates of change of different processes, the interdependence among these processes to be reflected in the correlation based on interindividual differences would be stronger in the older groups (i.e., an illustration of one variant of the dedifferentiation phenomenon).

While Hofer and Sliwinski's analyses regarding the NAC and SNAC designs are sound, without further explication (e.g., invoking additional assumptions) we do not see how such a time- (or life history-) dependent view of accumulating effects of rates of change would also capture the inverted phenomenon of ability differentiation during child development, as they suggested [see target article]. Furthermore, when SNAC designs are used, although other confounding sources of variance, in particularly mortality effects, would not directly affect the estimate of interdependence between variables $\mathrm{X}$ and $\mathrm{Y}$ through mean age trends as in the age-heterogeneous design, they still could exert indirect effects at the level of sampling.

\section{Age Is Not Aging, and Aging Itself Is Not the 'Clocks' That Time Aging}

There is another basic reason why it is worrisome if population mean age trends in cross-sectional age-heterogeneous samples are confounded in the inferences about the degree and interdependence of aging changes. In essence, a rigid metric of physical time (or chronological age here) may not be of direct relevance to the internal dynamic of many real-life physical and biological systems [4], an issue that has been confronting researchers of dynamic processes in general.

In the field of developmental and aging research, time and again researchers revisit Wohlwill's [5] earlier questioning of using chronological age as a proxy for developmental and aging effects on behavioral and cognitive changes. The problem is twofold: First, age is not an individual characteristic that can be experimentally manipulated; thus, at most, it is only a quasi-independent variable. Second, at best, age can only capture part of the direct functional relationships between sets of endogenous (and/or exogenous) conditions and the observed developmental and aging changes. It does not offer explanations. In other words, the 'cranks of aging' are turned by neurobiological and cultural-social mechanisms that themselves need to be directly examined. 
Facets of these two longstanding problems are reflected in Hofer and Sliwinski's concerns. Analytically, it is clear that correlations based on cross-sectional age differences may not reflect as much about patterns of causal relations between aging processes as the research practice of using statistical confirmatory mediation models of age-heterogeneous cross-sectional data implicitly assumes. Empirically, however, the severity of the implications derived from Hofer and Sliwinski's formal analyses may not be as general and prevalent across phenomena and studies. For instance, if in some case it is known from longitudinal outcomes that there are few individual differences in initial level and in the rates of change of some processes, simple age partial analyses would suffice to avoid the confound from mean age trends in the sample. More generally, depending on the extent of the mean age trend and the magnitudes of interindividual differences in the rate of change and initial level in a given variable, guidelines regarding how narrow the selected age range should be may be determined. Possible empirical variants notwithstanding, the analytical results and their implications of a pressing need for revising theoretical conceptualizations and methods for studying aging change cannot be denied.

The designs Hofer and Sliwinski proposed are useful in as much as they avoid confounding mean age trends with analyses of correlated aging changes. However, in order to understand the causes of aging and the dynamics of aging change, besides the NAC, SNAC, and longitudinal designs, other research methodologies along with more explicit theoretical conceptions are necessary. In the following we elaborate our views on these two aspects.

\section{A 'New' Call for Explicit Theories of Change: Revisiting Classical Process-Oriented Conceptions}

The seasoned researchers in the field might get a sense of déjà vue reading 'new' proposals to revisit the more process-oriented (i.e., time-ordered markers of endogenous or exogenous processes) rather than solely ageoriented conceptions of development and aging. The classical volume by Baltes and Nesselroade [6] pointed out that in some conceptions of development, chronological age is considered indirectly relevant or even irrelevant for identifying the underlying 'clocks' that time developmental processes [cf. 7]. The focus was, rather, on timerelated trajectories of behavioral and cognitive changes such as developmental progressions, epochs, stages, and general processes of acquisition and maintenance $[8,9]$.
Specifically, Wohlwill [5] in 1970 suggested that chronological age should be integrated into the dependent variable, rather than be treated as an independent variable in development research by specifying endogenous and (or) exogenous conditions and parameters that have direct functional relations with behavioral and cognitive changes observed over age. These earlier views were not put into practice at the time, because knowledge about what some of these parameters could be was scarce and methods to investigate them nonexistent.

After four decades, the search to unpack functional factors and mechanisms contributing to aging remains the prime task. In the current zeitgeist, however, a fair amount of information about biological [10], cognitive [11], and social [12] influences on aging has accumulated, and perhaps we are now in a better position to study some of these candidate factors more directly. This calls for more explicit theories of aging changes, specifying not only some of the 'clocks' that time aging, but also the mechanisms and processes by which the clocks run, and their expressed effects with respect to performance level and rates of change at the individual and between-individual levels. Here we speculate on two general considerations for further specifications of cognitive aging theories.

\section{Causes for Change and Causes for Interindividual Differences in Change}

It is emphasized that causes of intraindividual change may not entirely overlap with causes of interindividual differences in intraindividual change [6]. Not only do methods need to be developed to examine these aspects of variance more directly; theories need to be more specific in comparing, contrasting, and relating the causes of, and interindividual differences in, changes. Some confusing debates regarding mismatches between theories and the types of methods and data sought to test them, that is evident in the current literature on speed and other general accounts of cognitive aging, could be avoided, if theories were brought to the next level of specification. For instance, in the context of the target article, Hofer and Sliwinski's view is that the speed and common-cause accounts should be tested in terms of correlated rates of change in longitudinal studies. When lacking longitudinal data, NAC designs are better suited to test these hypotheses than age-heterogeneous designs, because they could more 'purely' reflect interindividual differences in the rates of change and the degree of their interdependence. We agree with the problem of confounding mean age trends with information reflecting correlated rates of 


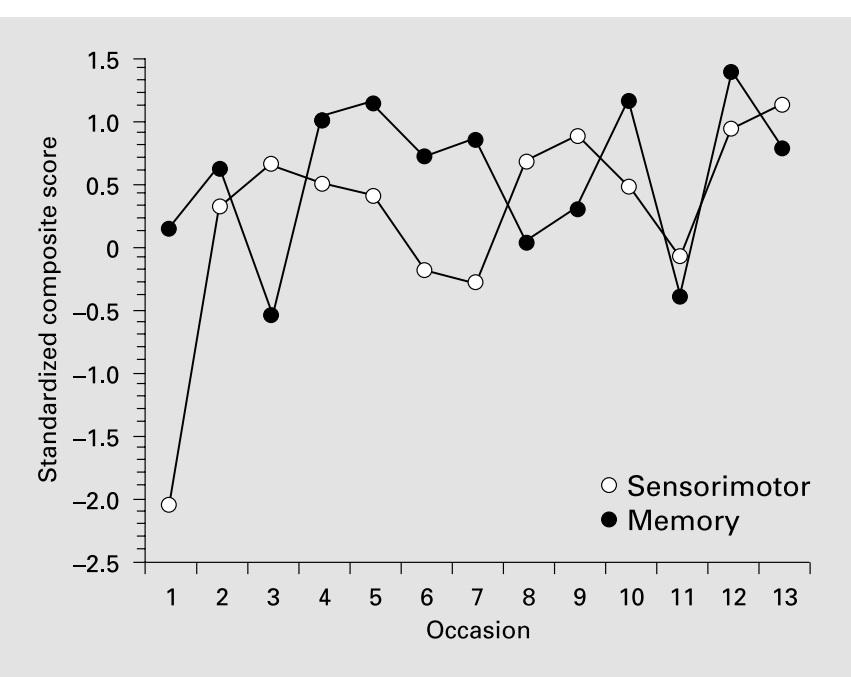

Fig. 2. Patterns of intraindividual fluctuation (short-term changes) in memory and sensorimotor performance in 1 old individual. The two trajectories show lead-lag patterns that need to be addressed by dynamic relations rather than static associations between rates of change as primarily discussed in the target article.

change using age differences obtained from age-heterogeneous samples. However, our reading of these accounts suggests that neither of them were specified with sufficient detail about (1) whether the proposed constructs should be viewed as causes for intraindividual changes or interindividual differences in rates of change or (2), if related, what roles the theoretical constructs play in bringing about such relations. Thus, these accounts need more specification before it can be decided whether correlated rates of change would be the best kind of evidence to test them.

\section{Constellation and Dynamics of Causes at Different Levels}

It is doubtful that polarized research efforts motivated solely to resolve the debate of whether aging is general or specific are the most fruitful approach to tackle questions about mechanisms of aging. Even if a few factors are more salient and influential than others [see 11 for a review], such factors 'turn their cranks' within the context of other influences from different levels and with 'working' mechanisms that are currently underspecified in most accounts. A more fruitful approach now would be to theorize in terms of the relations and mechanisms relating a constellation of these factors [cf. 10]. It may also be the case that different causes could be causally associated via processes leading to temporal cascade effects with some causes anteceding others temporally. Conceptualizations of both proximal and distal (i.e., time-lagged) effects need to be more centrally incorporated, as many contemporary theories do not suggest looking for dynamics. For instance, figure 2 plots biweekly short-term intraindividual fluctuations across 26 weeks in memory and sensorimotor measures of 1 old participant in the McArthur Successful Aging Studies [13]. The question here is not so much with respect to rates of change; the issue is more in terms of whether memory and sensorimotor performance fluctuate together. At first glance, one is likely to conclude, 'no'. On many occasions when memory performance shows improvement, sensorimotor performance is depressed and vice versa. However, in general, the two trajectories show a lead-lag pattern. The possibility that the two processes change in similar ways but with a time lag needs to be considered. Taken together, future theoretical advances need to be more specific about the different levels of time scale associated with changes (e.g., long-term changes or short-term fluctuations) and the patterns of expected relations between causes and effects that might be observed. Furthermore, in addition to considering temporal cascade effects, the notion of cascade effects across levels with possibly bidirectional influences between the genetic-neuronal level on the one hand and the cognitive and behavioral levels on the other hand need to be considered $[14,15]$.

\section{Others Alternative Methods and Paradigms}

Finally, we briefly highlight a few relatively recent methods and paradigms that may be useful in taking us into the next level of understanding aging processes. Regarding time- or process-oriented conceptions of aging, recently developed methods of marker or curve registration [4] and dynamic time warping in statistical data analyses [16] accord with proposals about identifying empirically or theoretically driven features of aging, and to study aging along these dimensions instead of chronological age. For instance, the transition from the third age into the fourth age or the transition into final terminal decline [17] varies across individuals with respect to chronological age. However, features of these transitions may be observable with respect to some biological and psychological processes. In this case, methods similar to dynamic time warping developed for functional data analysis may be applicable to align individual change trajectories and transform aging changes taking place on the chart of chro- 
nological age with respect to these identified features. To capture the dynamics of causal patterns between aging processes, recent methods of dynamic factor analyses [18] and latent-change score methods [19] have been developed to serve this purpose. Regarding considerations of a constellation of causes, rather than a single cause, of aging, structural equation models could be designed to capture both general and specific effects which allow for direct comparisons of these effects and their relations to other criterion processes and factors.

To consider the cascade of causes and effects across different levels, research paradigms and strategies of cognitive and computational neurosciences are of interest. Take, for example, the issues about the relation between sensorimotor and cognitive functioning in old age or the dedifferentiation phenomenon. Besides testing the existence of these effects with respect to correlated rates of change as suggested by Hofer and Sliwinski, empirical paradigms have been applied directly to examine how sensorimotor and cognitive processes trade off in dual task paradigms [20], in terms of neuronal activity in the sensorimotor cortex that is invoked by the act of remembering events [21], and how different processes become less lateralized in the aging brain $[22,23]$. Computational models capturing increased bilateral cortical information processing and exploring their relations to neuromodulatory mechanisms are under way [24]. There are also other recent computational and empirical investigations focusing on relating declines in neuromodulation, attentional control mechanisms, and cognitive aging [25]. More specifically, with respect to the design issues that are raised, computational neuroscience approaches have been ap- plied to generate groups of neural networks that vary in narrow ranges with respect to a parameter simulating an empirically motivated neuromodulatory mechanism $[26$, 27]. Such approaches approximate a computational analogue of Hofer and Sliwinski's SNAC design in conjunction with a process-oriented specification of relating some aspects of cognitive aging to neuromodulation.

\section{Conclusions}

Hofer and Sliwinski convince us about the problem of confounding mean age trends with information reflecting patterns of correlated rates of changes in age-heterogeneous cross-sectional designs. Faced with this, we echo classical theoretical positions on the somewhat 'superficial' and 'spurious' role of chronological age in aging and developmental research. Maybe it is time now to once more revisit earlier calls for process-oriented rather than age-oriented conceptions of development and aging. With new statistical, computational, and experimental advances at different levels at hand, maybe the field can now take a step forward to probe into 'the ticking of the clocks that time aging'.

\section{Acknowledgments}

We thank Paul B. Baltes, Paolo Ghisletta, Tania Singer, Jacqui Smith, and Paul Verhaeghen for valuable comments and suggestions on an earlier draft of this commentary. This research was supported by the research funds of the Max Planck Society.

\section{References}

1 Schaie WK: A general model for the study of developmental problems. Psychol Bull 1965; 64:92-107.

2 Verhaeghen P, Salthouse TA: Meta-analyses of age-cognition relations in adulthood: Estimates of linear and non-linear age effects and structural models. Psychol Bull 1997;122:231-249.

3 Schaie WK: Intellectual Development in Adulthood: The Seattle Longitudinal Study. Cambridge, Cambridge University Press, 1996.

4 Ramsay JO, Li X: Curve registration. J R Stat Soc 1998;60:351-363.

5 Wohlwill JF: The age variable in psychological research. Psychol Rev 1970;77:49-64.
6 Baltes PB, Nesselroade JR: History and rationale of longitudinal research; in Nesselroade JR, Baltes PB (eds): Longitudinal Research in the Study of Behavior and Development. New York, Academic Press, 1978, pp 1-29.

7 Featherman DL, Petersen T: Makers of aging: Modeling the clocks that time us. Res Aging 1986;8:339-365.

8 Gagné RM: Contributions of learning to human development. Psychol Rev 1968;75:177191.

9 Baer DM: An age-irrelevant concept of development. Merrill Palmer Q 1970;16:238-246.

10 McClearn GE: Biomarkers of age and aging. Exp Gerontol 1997;32:87-94.

11 Craik FIM, Salthouse TA (eds): The Handbook of Aging and Cognition, ed 2. Mahwah, Erlbaum, 2000 .
12 Baltes MM: The Many Faces of Dependency in Old Age. New York, Cambridge University Press, 1996.

13 Li S-C, Aggen SH, Nesselroade JR, Baltes PB: Short-term fluctuations in elderly people's sensorimotor functioning predict text and spatial memory performance: The MacArthur Successful Aging Studies. Gerontology 2001;47: 100-116.

14 Gottlieb G: Normally occurring environmental and behavioral influences on gene activity: From central dogma to probabilistic epigenesis. Psychol Rev 1998; 105:792-802.

15 Li S-C: Connecting the many levels and facets of cognitive aging. Curr Dir Psychol Sci 2002; 11:38-43. 
16 Ramsay JO, Silverman BW: Functional Data Analysis. New York, Springer, 1997.

17 Baltes PB, Smith J: Multilevel and systemic analyses of old age: Theoretical and empirical evidence for a fourth age; in Bengtson VL, Schaie KW (eds): Handbook of Theories of Aging. New York, Springer, 1999, pp 153173.

18 Nesselroade JR, Molenaar P: Pooling lagged covariance structures based on short, multivariate time series for dynamic factor analysis; in Hoyle R (ed): Research Strategies for Small Samples. Newbury Park, Sage, 2000.

19 McArdle JJ: A latent difference score approach to longitudinal dynamic structural analyses; in Cudeck R, du Toit S, Sorbom D (eds): Structural Equation Modeling: Present and Future. Lincolnwood, Scientific Software International, 2000, pp 342-380.
20 Li KZ, Lindenberger U, Freund AM, Baltes PB: Walking while memorizing: Age-related differences in compensatory behavior. Psychol Sci 2001;19:230-237.

21 Nilsson LG, Nyberg L, Klingberg T, Aberg C, Persson J, Roland PE: Activity in motor areas while remembering action events. Neuroreport 2000;11:2199-2201.

22 Reuter-Lorenz PA, Jonides J, Smith EE, Hartley A, Miller A, Marshuetz C, Koeppe RA: Age differences in the frontal lateralization of verbal and spatial working memory revealed by PET. J Cogn Neurosci 2000;12:174-187.

23 Cabeza R, McIntosh AR, Tulving E, Nyberg L, Grady CL: Age-related differences in effective neural connectivity during encoding and recall. Neuroreport 1997;8:3479-3483.

24 Li S-C, Sikström S: Computational links between neuromodulation, representation, distinctiveness, memory and temporal capacities in aging. 3rd Int Conf on Memory, Valencia 2001.
25 Braver TS, Deanna MB, Keys BA, Carter CS, Cohen JO, Kaye JA, Janowsky JS, Taylor SF, Yesavage JA, Mumenthaler MS, Jagust WJ, Reed BR: Context processing in older adults: Evidence for a theory relating cognitive control to neurobiology in healthy aging. J Exp Psychol Gen 2001;130:746-763.

26 Li S-C, Lindenberger U: Cross-level unification: A computational exploration of the link between deterioration of neurotransmitter systems and dedifferentiation of cognitive abilities in old age; in Nilsson LG, Markowitsch M (eds): Cognitive Neurosciences of Memory. Toronto, Hogrefe \& Huber, 1999, pp 104-146.

27 Li S-C, Lindenberger U, Frensch PA: Unifying cognitive aging: From neuromodulation to representation to cognition. Neurocomputing 2000;32/33:879-890. 